\title{
Editorial
}

\section{Chaos-Fractals Theories and Applications}

\author{
Hai Yu, ${ }^{1}$ Ahmed Elwakil, ${ }^{2}$ Lev Ryashko, ${ }^{3}$ Yuncai Wang, ${ }^{4}$ and Guanrong (Ron) Chen ${ }^{5}$ \\ ${ }^{1}$ Software College, Northeastern University, Shenyang 110819, China \\ ${ }^{2}$ Department of Electrical and Computer Engineering, University of Sharjah, Sharjah 27272, United Arab Emirates \\ ${ }^{3}$ Institute of Mathematics and Computer Sciences, Ural Federal University, Lenina 51, Ekaterinburg, Russia \\ ${ }^{4}$ College of Physics and Optoelectronics, Taiyuan University of Technology, Taiyuan 030024, China \\ ${ }^{5}$ Department of Electronic Engineering, City University of Hong Kong, 83 Tat Chee Avenue, Kowloon, Hong Kong \\ Correspondence should be addressed to Hai Yu; yuhai@mail.neu.edu.cn
}

Received 26 February 2014; Accepted 26 February 2014; Published 2 April 2014

Copyright (C) 2014 Hai Yu et al. This is an open access article distributed under the Creative Commons Attribution License, which permits unrestricted use, distribution, and reproduction in any medium, provided the original work is properly cited.

Chaos-fractals theories and applications play an important role in nonlinear science research. The subject has been widely investigated with significant progress and achievements especially in recent years. In addition, it has been applied to many scientific disciplines, such as meteorology, physics, engineering, economics, biology, and even philosophy. This special issue on Chaos-Fractals Theories and Applications publishes 19 papers, most of which are carefully reviewed by experts and peers in the field.

Some research on chaos theory is addressed by 5 papers. For example, T. Lu et al. study the retentivity of chaos under topological conjugation, proving that chaotic properties are all preserved under such a condition. However, an example is given to show that $\mathrm{Li}$-Yorke chaos is not preserved under topological conjugation if the domain is extended to a general metric space. J. Luo suggests a state-feedback control method for fractional-order nonlinear systems subject to input saturation. It is shown that linear state-feedback controller can be used to control the fractional-order nonlinear systems.

There are 6 papers focusing on fractals research. For instance, M. Romera et al. introduce a method to draw complete external rays based on the escape lines and Bézier curves. It solves the limitation in drawing an external ray because it reaches a point from which the drawing tool cannot continue to work. J. Lu et al. develop a fractal color image watermarking method. S. Zhu et al. explore an efficient fractal video codec for compressing multiviews. They make full use of the characteristic of fractal video coding and the nature of video particularly.
In addition, there are 8 papers which address chaosfractals applications. Just to introduce a few, T. Zhao et al. report their recent works on free space ranging with chaotic light. Using a laser diode with optical feedback as a chaotic source, a prototype of chaotic lidar was developed, which can achieve a range-independent resolution of $18 \mathrm{~cm}$ and measurable distance of $130 \mathrm{~m}$ in distance. $\mathrm{Y}$. Wu et al. report their investigation on the time delay signature in chaotic semiconductors, subject to delayed filtered optical feedback, of which a Fabry-Pérot interferometer type of filter is placed in the external cavity. Especially, a new memristor based chaotic circuit is presented by Y. Li et al. It is generated by replacing the nonlinear resistor in Chua's circuit with a fluxcontrolled memristor and a negative conductor. The dynamical behaviors are verified by both computer simulations and laboratory experiments.

By filing these papers together in a special issue, we wish to provide better views for our readers and researchers about the important areas of chaos-fractals research, regarding both theory and applications.

Hai Yu

Ahmed Elwakil

Lev Ryashko

Yuncai Wang

Guanrong (Ron) Chen 


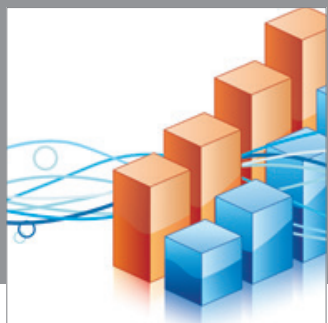

Advances in

Operations Research

mansans

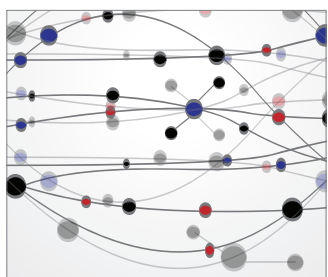

The Scientific World Journal
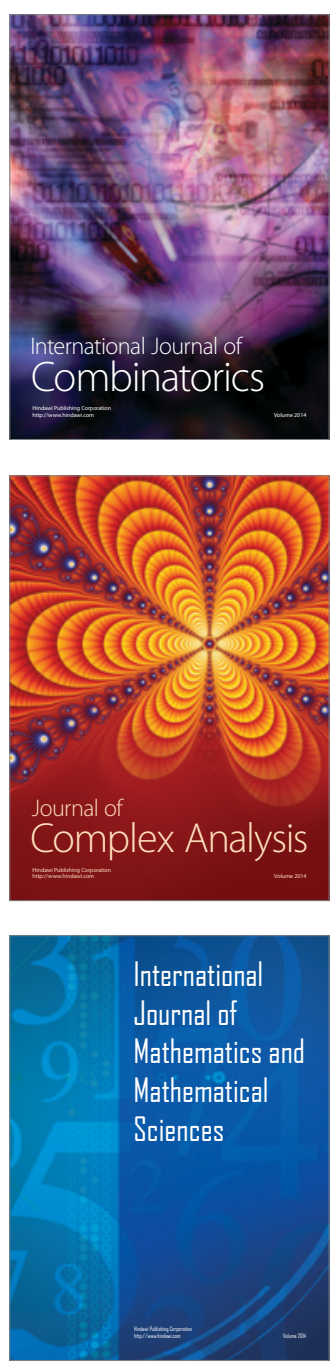
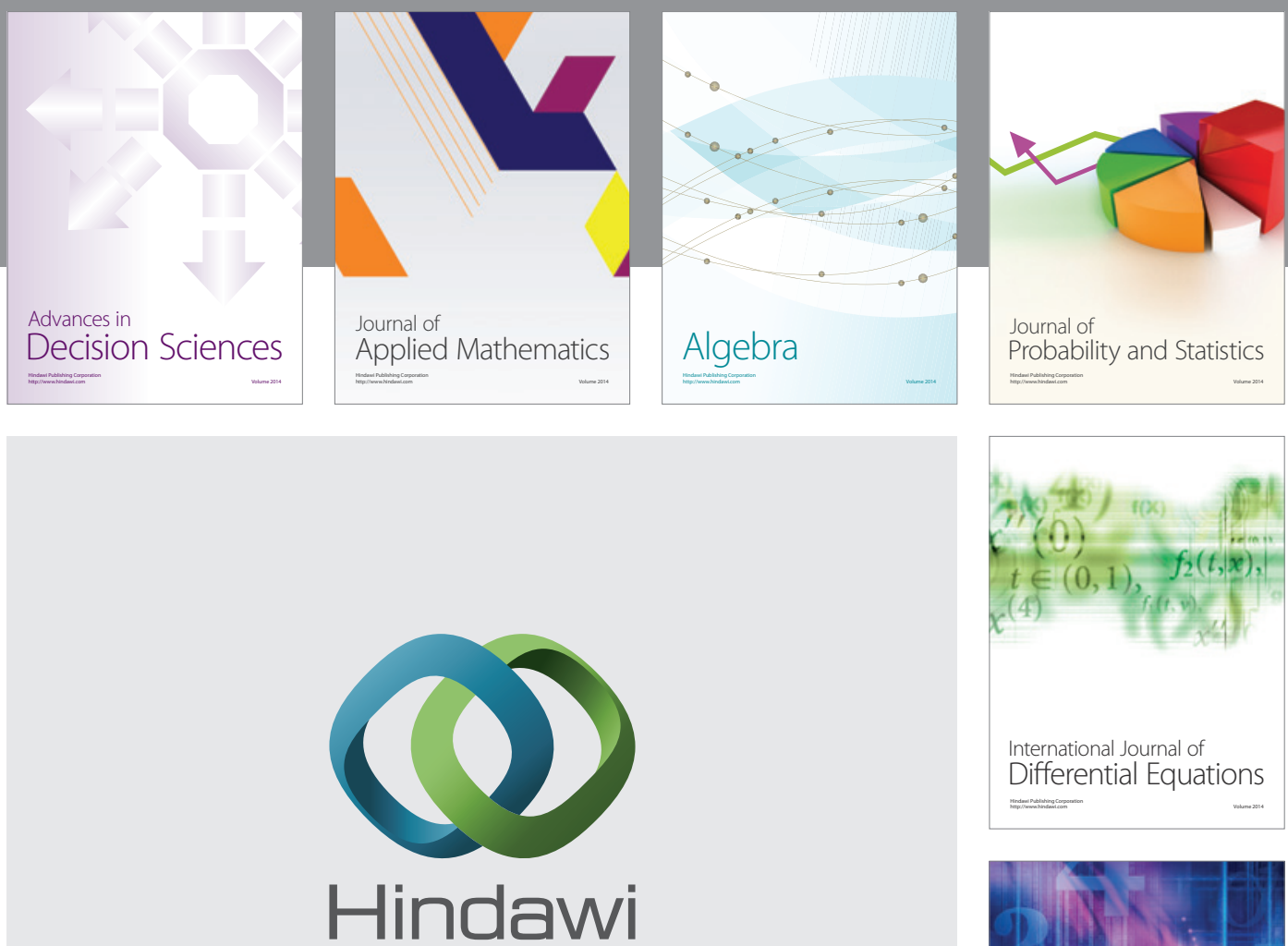

Submit your manuscripts at http://www.hindawi.com
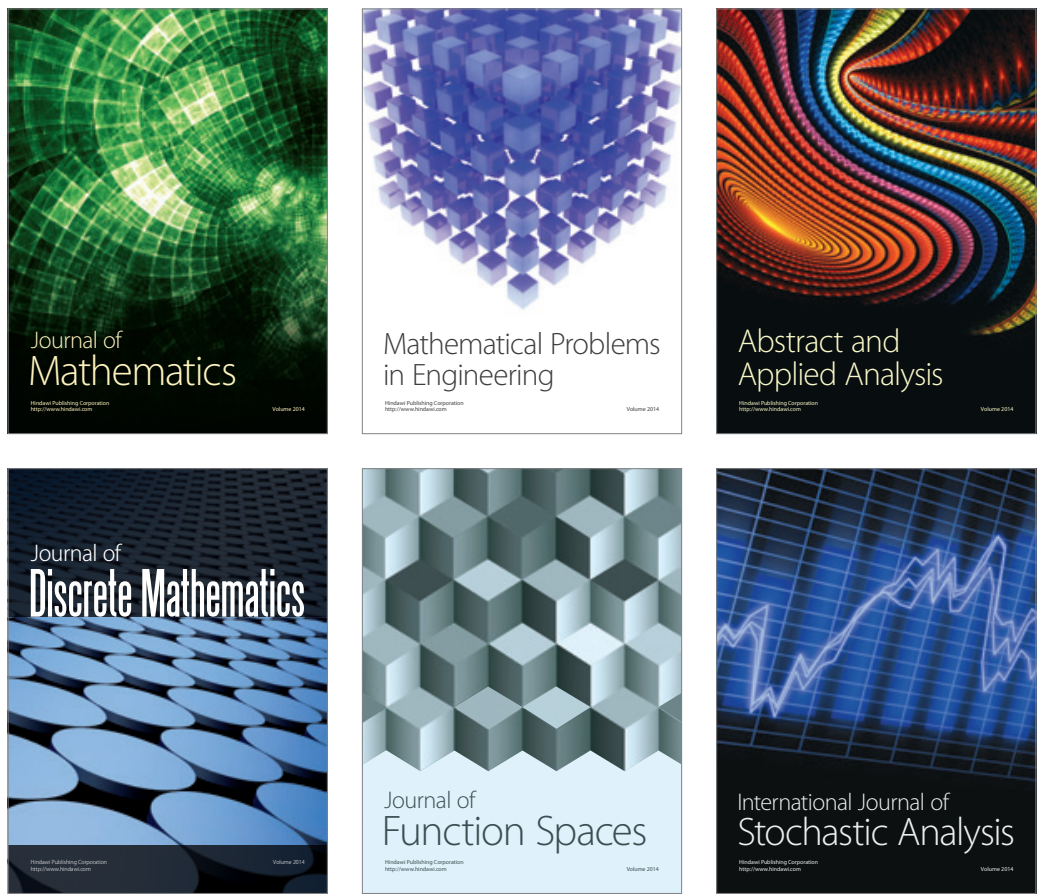

Journal of

Function Spaces

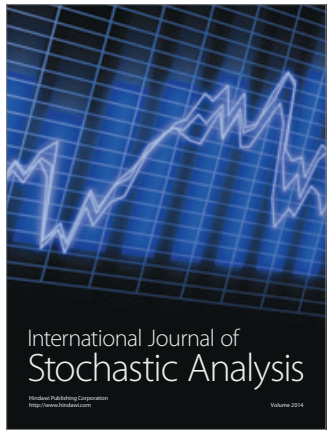

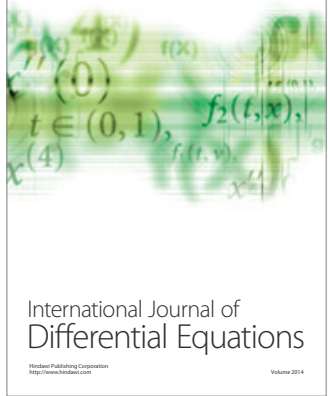
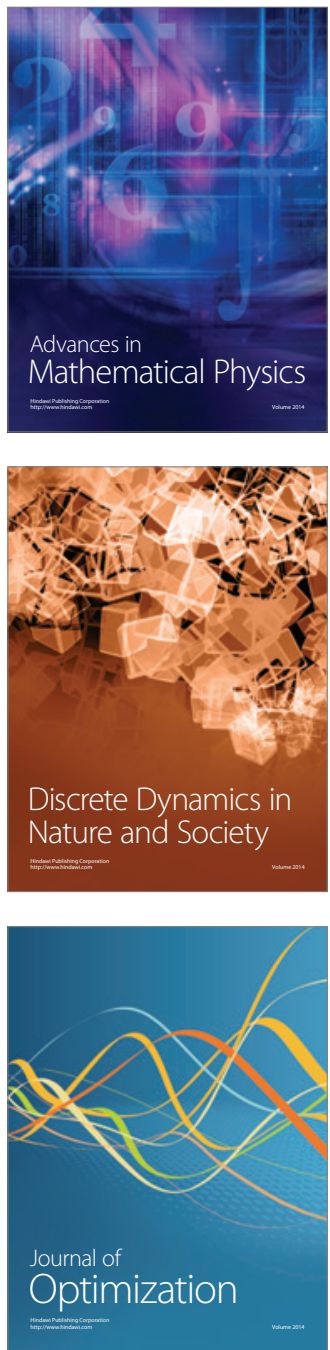